\title{
A NOTE ON NORMAL-OPERATOR-VALUED ANALYTIC FUNCTIONS ${ }^{1}$
}

\author{
J. GLOBEVNIK AND I. VIDAV
}

ABSTRACr. In this paper we prove that the set of values of a normal-operator-valued function, defined and analytic on an open connected set in the complex plane, is commutative.

The aim of this note is to prove that the set of values of a normaloperator-valued function, defined and analytic on an open connected set in the complex plane, is commutative. We were not able to find whether such a result is already known.

Throughout the paper we denote by $\mathscr{N}$ the set of nonnegative integers and by $L(X)$ the $C^{*}$-algebra of all bounded endomorphisms of a complex Hilbert space $X$.

LEMMA. Let $X$ be a complex Hilbert space and $\mathscr{D}$ the open unit disc in the complex plane. Let $F: \mathscr{D} \rightarrow L(X)$ be a normal-operator-valued analytic function given by

$$
F(\zeta)=A_{0}+A_{1} \zeta+A_{2} \zeta^{2}+\cdots \quad(\zeta \in \mathscr{D}) .
$$

Then

$$
\begin{array}{cc}
A_{i} A_{j}=A_{j} A_{i} & (i \in \mathscr{N}, j \in \mathscr{N}), \\
A_{i} A_{j}^{*}=A_{j}^{*} A_{i} & (i \in \mathscr{N}, j \in \mathscr{N}) .
\end{array}
$$

Proof. By the normality assumption we have

$$
F(\zeta) F(\zeta)^{*}-F(\zeta)^{*} F(\zeta)=0 \quad(\zeta \in \mathscr{D}) .
$$

Since $F$ is analytic on $\mathscr{D}$, the series

$$
\sum_{i=0}^{\infty}\left\|A_{i}\right\| r^{i}
$$

converges for $0 \leqq r<1$ (cf. [2, Theorem 3.11.4]). This convergence and the isometricity of involution allow term-by-term multiplication in (2)

Received by the editors June 26, 1972.

AMS (MOS) subject classifications (1970). Primary 30A96.

1 This work was supported by the Boris Kidrič Fund.

(c) American Mathematical Society 1973 
which gives

$$
\sum_{i=0}^{\infty}\left[\sum_{k=0}^{i}\left(A_{k} A_{i-k}^{*}-A_{i-k}^{*} A_{k}\right) \zeta^{k} \bar{\zeta}^{i-k}\right]=0 \quad(\zeta \in \mathscr{D}) .
$$

Since the series (3) converges for $0 \leqq r<1$ it is easy to see that also the series

$$
\sum_{i=0}^{\infty}\left[\sum_{k=0}^{i}\left\|A_{k} A_{i-k}^{*}-A_{i-k}^{*} A_{k}\right\|\right] r^{i}
$$

converges for $0 \leqq r<1$. It follows that the series (4) converges uniformly on every closed disc contained in $\mathscr{D}$.

Let $p \in \mathscr{N}$. Multiplying (4) with $\zeta^{p-1}$ and integrating term-by-term along the boundary of the disc with radius $r<1$ and with center at the point 0 we obtain

$$
\sum_{i=0}^{\infty}\left(A_{i} A_{i+p}^{*}-A_{i+p}^{*} A_{i}\right) r^{2(i+p)}=0 \quad(0 \leqq r<1) .
$$

The convergence of the series (5) implies that the series

$$
G(\zeta)=\sum_{i=0}^{\infty}\left(A_{i} A_{i+p}^{*}-A_{i+p}^{*} A_{i}\right) \zeta^{2(i+p)}
$$

is absolutely convergent on $\mathscr{D}$. This means that the function $\zeta \mapsto G(\zeta)$ is analytic on $\mathscr{D}$. Further, (6) tells us that $G(r)=0(0 \leqq r<1)$. By a wellknown theorem it follows that $G(\zeta)=0(\zeta \in \mathscr{D})$, which implies

$$
A_{i} A_{i+p}^{*}-A_{i+p}^{*} A_{i}=0 \quad(i \in \mathscr{N}) .
$$

Since $p \in \mathscr{N}$ was arbitrary it follows that

$$
A_{i} A_{j}^{*}=A_{j}^{*} A_{i} \quad(i \in \mathscr{N}, j \in \mathscr{N}) .
$$

In particular, (7) tells that $A_{i}(i \in \mathcal{N})$ are normal operators. Now by the theorem of Fuglede (cf. [1, p. 934]), (7) implies $A_{i} A_{j}=A_{i} A_{i}(i \in \mathscr{N}$, $j \in \mathscr{N}$ ), which, together with (7), proves the assertion. Q.E.D.

THEOREM. Let $X$ be a complex Hilbert space, $\mathscr{D}$ an open connected set in the complex plane and let $F: \mathscr{D} \rightarrow L(X)$ be an analytic function. Let a neighborhood $\mathscr{U}\left(\zeta_{0}\right)$ of a point $\zeta_{0} \in \mathscr{D}$ exist such that $F(\zeta)\left(\zeta \in \mathscr{U}\left(\zeta_{0}\right)\right)$ are normal operators. Then $F(\zeta)$ is a normal operator for every $\zeta \in \mathscr{D}$ and

$$
F\left(\zeta_{1}\right) F\left(\zeta_{2}\right)=F\left(\zeta_{2}\right) F\left(\zeta_{1}\right) \quad\left(\zeta_{1} \in \mathscr{D}, \zeta_{2} \in \mathscr{D}\right) .
$$

Proof. With no loss of generality we may assume that $\mathscr{U}\left(\zeta_{0}\right)$ is an open disc with radius $r$ and with center at the point $\zeta_{0}$. Expand $F$ into the 
Taylor series

$$
F(\zeta)=A_{0}+A_{1}\left(\zeta-\zeta_{0}\right)+A_{2}\left(\zeta-\zeta_{0}\right)^{2}+\cdots .
$$

Clearly we may assume that $\zeta_{0}=0$ and $r=1$, and by the Lemma we get

$$
\begin{array}{ll}
A_{i} A_{j}=A_{j} A_{i} & (i \in \mathscr{N}, j \in \mathscr{N}), \\
A_{i} A_{j}^{*}=A_{j}^{*} A_{i} & (i \in \mathscr{N}, j \in \mathscr{N}) .
\end{array}
$$

This assures (cf. [3, p. 182]) the existence of a commutative $C^{*}$-subalgebra $\mathscr{A}$ of the algebra $L(X)$ which contains the operators $A_{i}(i \in \mathscr{N})$. Since $\mathscr{A}$ is a closed linear subspace of $L(X)$, by the Hahn-Banach theorem a set $\mathscr{T}$ of bounded linear functionals on $L(X)$ exists such that $A \in \mathscr{A}$ if and only if $u(A)=0(u \in \mathscr{T})$. Now, clearly $F(\zeta) \in \mathscr{A}\left(\zeta \in \mathscr{U}\left(\zeta_{0}\right)\right)$ so that $u[F(\zeta)]=0$ $\left(\zeta \in \mathscr{U}\left(\zeta_{0}\right), u \in \mathscr{T}\right)$. By the analytic continuation principle the connectedness of $\mathscr{D}$ implies that $u[F(\zeta)]=0(\zeta \in \mathscr{D}, u \in \mathscr{T})$ which means that $F(\zeta) \in \mathscr{A}(\zeta \in \mathscr{D})$. Since $\mathscr{A}$ is a commutative $C^{*}$-subalgebra of $L(X)$, the assertion is proved. Q.E.D.

\section{REFERENCES}

1. N. Dunford and J. T. Schwartz, Linear operators. II: Spectral theory. Selfadjoint operators in Hilbert space, Interscience, New York, 1963. MR 32 \#6181.

2. E. Hille and R. S. Phillips, Functional analysis and semi-groups, rev. ed., Amer. Math. Soc. Colloq. Publ., vol. 31, Amer. Math. Soc., Providence, R.I., 1957. MR 19, 664.

3. C. E. Rickart, General theory of Banach algebras, University Series in Higher Math., Van Nostrand, Princeton, N.J., 1960. MR 22 \#5903.

Institute for Mathematics, Physics and Mechanics, University of LuUbljana, LuUbLJANA, YugoslaVia 